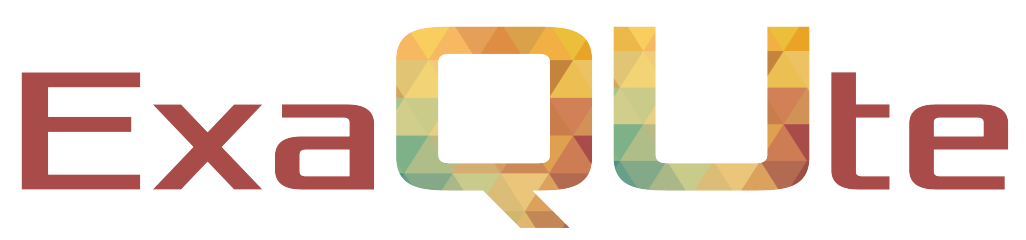

Exascale Quantification of Uncertainties for

Technology and Science Simulation

\title{
D4.1 Working Python API to schedule MLC routines
}

\section{Document information table}

\begin{tabular}{|l|l|}
\hline Contract number: & 800898 \\
\hline Project acronym: & ExaQUte \\
\hline Project Coordinator: & CIMNE \\
\hline Document Responsible Partner: & CIMNE \\
\hline Deliverable Type: & Software \\
\hline Dissemination Level: & Public \\
\hline Related WP \& Task: & WP 4, Task 4.1 \\
\hline Status: & Final version \\
\hline
\end{tabular}

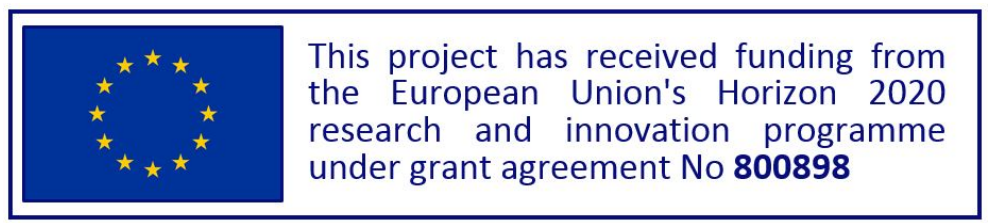




\section{Authoring}

\begin{tabular}{|c|c|c|c|c|}
\hline \multicolumn{5}{|l|}{ Prepared by: } \\
\hline Authors & Partner & Modified Page/Sections & Version & Comments \\
\hline Ramon Amela & $\mathrm{BSC}$ & & V0 & $\begin{array}{l}\text { Creation of the docu- } \\
\text { ment }\end{array}$ \\
\hline Rosa M. Badia & $\mathrm{BSC}$ & Sections $1,2,3$ and 5 & V0 & $\begin{array}{l}\text { Changes in some sec- } \\
\text { tions }\end{array}$ \\
\hline Riccardo Rossi & CIMNE & & & \\
\hline Stanislav Bohm & IT4i & & & \\
\hline Jakub Beranek & IT4i & & & \\
\hline Contributors & & & & \\
\hline & & & & \\
\hline 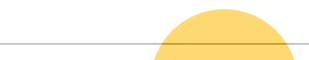 & & & & \\
\hline
\end{tabular}

\section{Change Log}

\section{\begin{tabular}{|l|l|l} 
Versions & Modified Page/Sections & Comments
\end{tabular}}
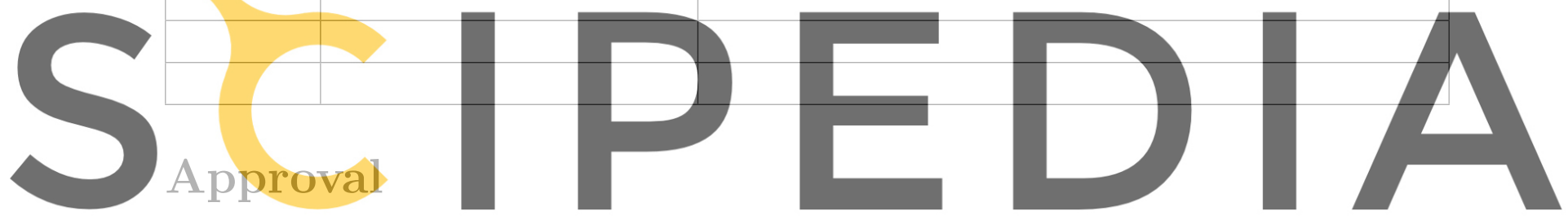

Register for free at hittps/dwww.scipedia.com to download the version without the y/atermark

\begin{tabular}{|l|l|l|l|l|}
\hline Task leader & Rosa M. Badia & BSC & 27.7 .18 & OK \\
\hline WP leader & Rosa M. Badia & BSC & 27.7 .18 & OK \\
\hline Coordinator & Riccardo Rossi & CIMNE & 27.7 .18 & OK \\
\hline
\end{tabular}




\section{Executive summary}

This deliverable focuses on the definition of a common API for the PyCOMPSs programming model and HyperLoom scheduler provided respectively by BSC and IT4I. The objective of the work is to hide the details of the actual task scheduling technology, so that the Multi Level Monte Carlo Python engine is agnostic of the backend being employed.

It includes the description of:

- Common API for calls

- Examples of usage

- Basic Documentation

The document also contains an initial description on how MPI-distributed data shall be treated from the scheduling point of view.
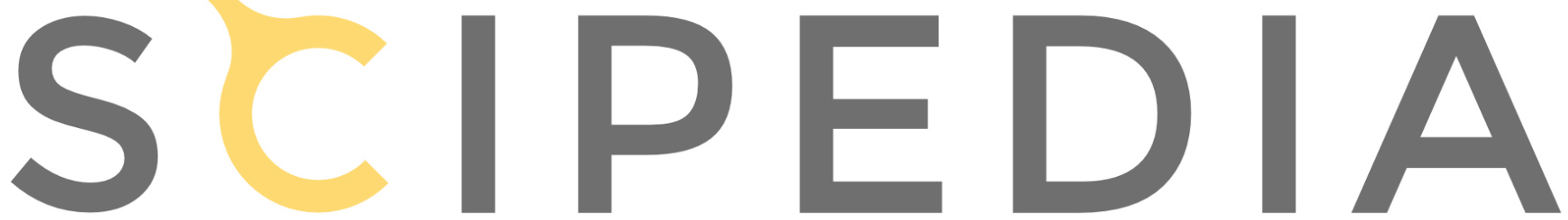

Register for free at https//www.scipedia.com to download the version without the watermark 


\section{Table of contents}

1 Introduction $\quad 8$

2 API definition $\quad 8$

2.1 Common API . . . . . . . . . . . . . . . . . . 8

2.1 .1 Launch process . . . . . . . . . . . . . . 8

2.1 .2 Distribution strategy . . . . . . . . . . . . . . . 8

2.1 .3 API format . . . . . . . . . . . . . . 8

2.1 .4 API calls . . . . . . . . . . . . . . . . . 9

2.1 .5 Task constraints . . . . . . . . . . . . . . . . . 9

2.2 PyCOMPSs functionalities . . . . . . . . . . . . . . . . . 9

$2.2 .1 \quad$ Files as a special type . . . . . . . . . . . . . . . . 9

2.2 .2 Constraint decorator ... . . . . . . . . . . . . . . 9

2.2 .3 Implement decorator . . . . . . . . . . . . . . . . . . . . . . . 12

2.3 HyperLoom functionalities . . . . . . . . . . . . . . . . . 12

2.3.1 Dynamic resource requests . . . . . . . . . . . . . . 12

2.3.2 Run external program with zero-copy execution . . . . . . . . . 13

3 Methods
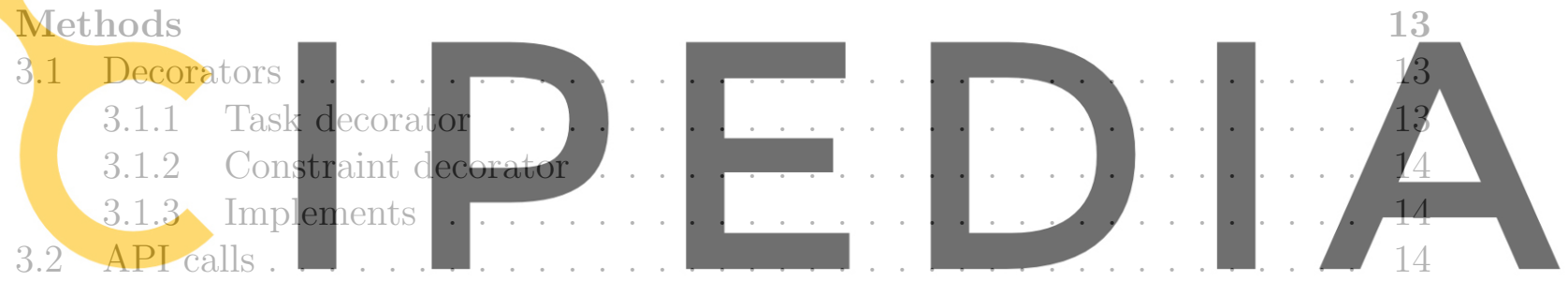

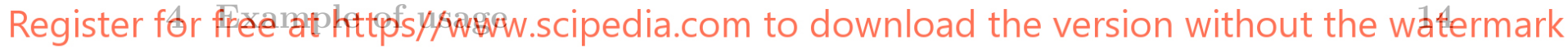

5 Data requirements 15

5.1 Single serialized object . . . . . . . . . . . . . . . . . 15

5.2 Distributed serialized object . . . . . . . . . . . . . . . . 15

$\begin{array}{ll}\text { A Methods } & 17\end{array}$

$\begin{array}{ll}\text { B Example of usage } & 17\end{array}$ 


\section{List of Figures}

1 Constraint basic usage . . . . . . . . . . . . . . . . . . . 10

2 Constraint advanced usage . . . . . . . . . . . . . . . . . 10

3 Implement usage example . . . . . . . . . . . . . . . . . . . . . 13

4 Decorator definition for the local case . . . . . . . . . . . . . . 17

5 API calls definition for the local case . . . . . . . . . . . . . 17

6 Basic use example . . . . . . . . . . . . . . . . . . . . . . . 18

$7 \quad$ Monte Carlo step definition . . . . . . . . . . . . . . . . . . . . 19

8 Model definition . . . . . . . . . . . . . . . . . . . . . . . 19

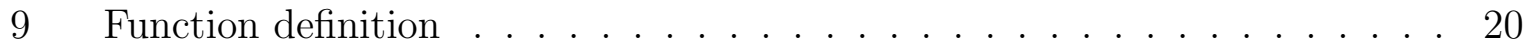
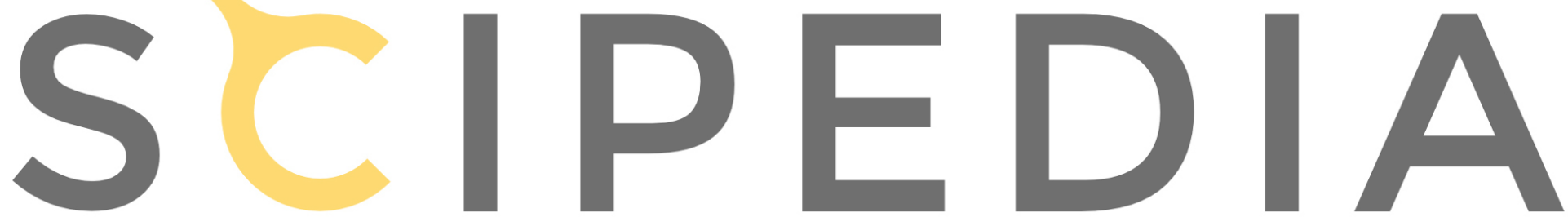

Register for free at https//www.scipedia.com to download the version without the watermark 


\section{List of Tables}

1 Arguments of the @constraint decorator ................ 11

2 Arguments of the @Processor decorator . . . . . . . . . . . . . . 12

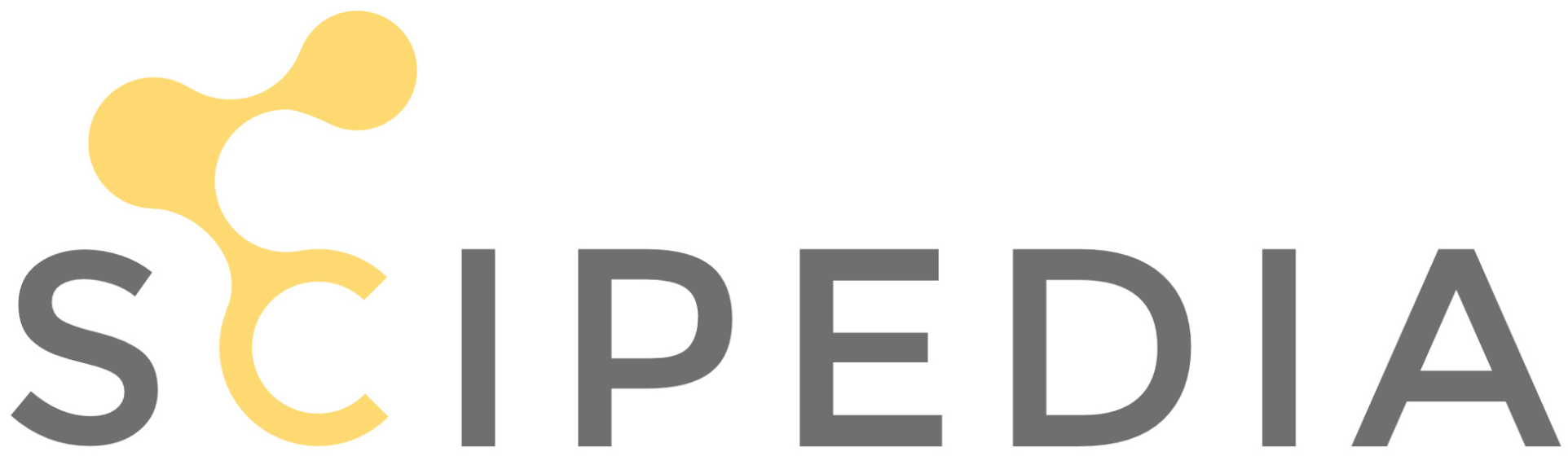

Register for free at https//www.scipedia.com to download the version without the watermark 


\section{Nomenclature / Acronym list}

\begin{tabular}{|l|l|}
\hline Acronym & Meaning \\
\hline API & Application Programming Interface \\
\hline ExaQUte & $\begin{array}{l}\text { EXAscale Quantification of Uncertainties for Technology } \\
\text { and Science Simulation } \\
\text { DArected Acyclic Graph }\end{array}$ \\
\hline FILE_IN & Path to a file passed to a function that is not modified \\
\hline FILE_INOUT & Path to a file passed to a function that is modified during the call \\
\hline FILE_OUT & Path to a file passed to a function that is created during the call \\
\hline HPC & High Performance Computing \\
\hline IN & Parameter of a function that is not modified \\
\hline INOUT & Parameter of a function that is modified during the call \\
\hline OpenMP & Open Multi Processing \\
\hline MPI & $\begin{array}{l}\text { Message Passing Interface } \\
\text { PBS }\end{array}$ \\
\hline PyCOMPSs & Python binding for COMPS Superscalar \\
\hline SLURM & Simple Linux Utility for Resource Management \\
\hline
\end{tabular}
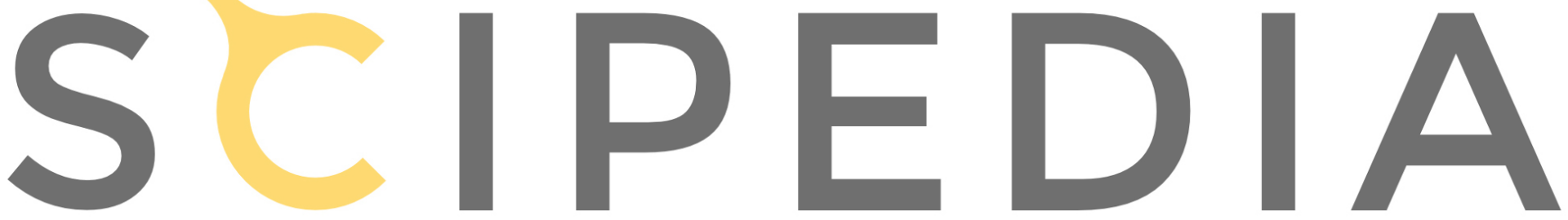

Register for free at https//www.scipedia.com to download the version without the watermark 


\section{Introduction}

PyCOMPSs and HyperLoom are task-based programming environment which enable the parallelization of sequential codes that can be then executed in distributed computing platforms.

State of the art description including references to relevant articles, e.g. [? ? ? ? ? ]

\section{API definition}

The API has been designed in such a way that it is able to express the union of functionalities supported by both PyCOMPSs and HyperLoom. The aim of this section is to explain the API desirable properties and show the reasoning behind the definitions.

\subsection{Common API}

First of all, both frameworks has been analyzed to find the common points. All along the discussion, it has been taken into account that the users intended to use the API are not computer scientists. Hence, simplicity has been taken as a capital property to be achieved. In addition, the design tries to make as easy as possible the transformation of a sequential code into a distributed one.
2.1.1 Launch process
Both BSC and IT4 have already develop
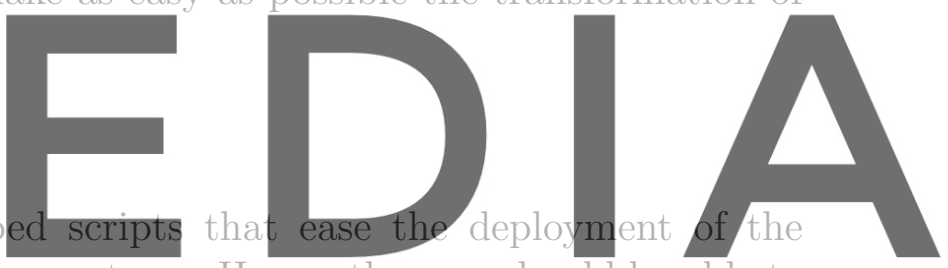

applications in HPC environments with queue systems. Hence, the user should be able to

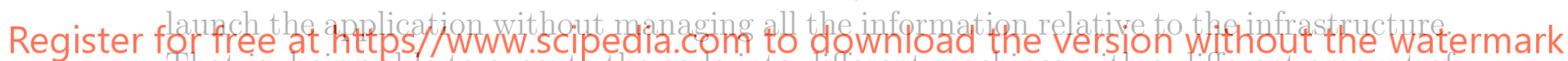
That is, being able to execute the code into different machines with a different amount of resources and a different architecture without changing it.

\subsubsection{Distribution strategy}

There are several distribution strategies. Nevertheless, both programming models define a workflow based on the taskification of the work. This means that some regions of code are defined to be executed remotely. The scheduler automatically detects the dependencies between tasks building the DAG that define the order in which the different tasks must be executed. In addition, generated objects are only brought to the master under demand.

\subsubsection{API format}

Considered the previous point, it has been decided to define the distributed portions of code with decorators. This strategy has several advantages. First of all, allows the user to define the main workflow sequentially, using a local definition of the decorator that avoids distributing the work. In addition, hides all the initialization process and avoids the direct communication between the user and the scheduler. Finally, makes possible to change from a sequential version to a distributed one o between both schedulers just changing the module import. This fact guarantees that the user can change the execution mode without modifying a single line of the code. 


\subsubsection{API calls}

The most important functionalities shared between both programming models are the following ones:

- Task definition

- Get value to the master/client node

- Wait until all the tasks have finished their execution

- Delete object from the remote nodes

\subsubsection{Task constraints}

Both PyCOMPSs and HyperLoom allow the user to specify resource constraints. PyCOMPSs has a wider variety of resource characteristics that can be indicated by the user. On the other side, HyperLoom allows the user to define them inline a dynamic way.

\subsection{PyCOMPSs functionalities}
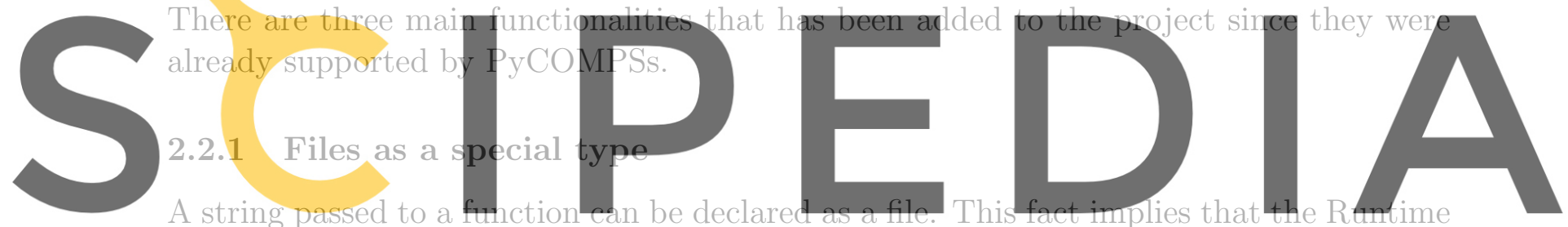

copies the corresponding file to/between/from the worker nodes to ensure that a given

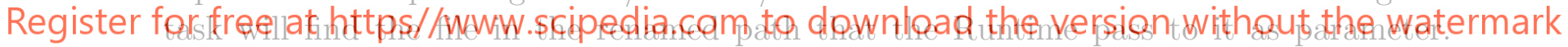

The same behavior than in the object case is offered, that is FILE_IN and FILE_INOUT.

In addition, it is possible to indicate FILE_OUT in case the file is generated into the task. This behavior does not make sense in the object case since either a reference to an already created object is passed to the function or a new instance is created and given back to the main program as return parameter. Finally, it has to be considered that this separate consideration implies the creation of the special call delete_file to delete all the available copies in the worker nodes.

\subsubsection{Constraint decorator}

It is possible to define constraints for each task. To this end, the decorator @ constraint followed by the desired constraints needs to be placed over the @task decorator as shown in Figure 1.

This decorator enables the user to set the particular constraints for each task, such as the amount of Cores required explicitly. Alternatively, it is also possible to indicate that the value of a constraint is specified in a environment variable. Figure 2 shows how to express this constraints.

A full description of the supported constraints can be found in Table 1. 

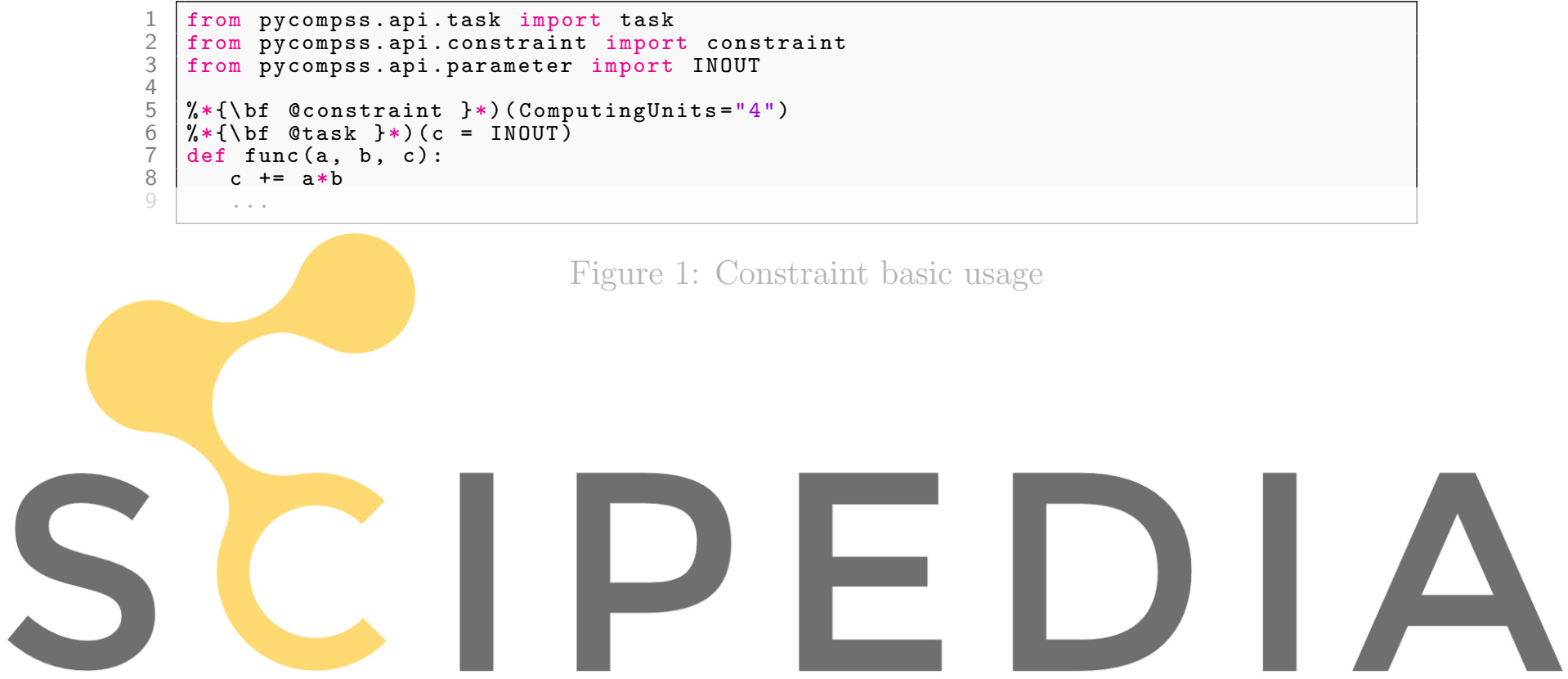

Register for free at https//www.scipedia.com to download the version without the watermark

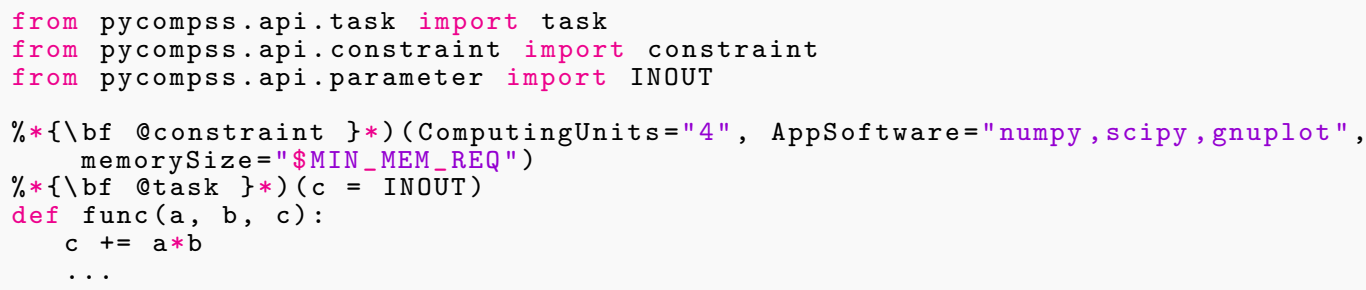

Figure 2: Constraint advanced usage 


\begin{tabular}{|c|c|c|c|}
\hline Field & Value type & Default value & Description \\
\hline ComputingUnits & $<$ string $>$ & ${ }^{*} 1 *$ & Required number of computing units \\
\hline ProcessorName & $<$ string $>$ & ".[unassigned]". & Required processor name \\
\hline ProcessorSpeed & $<$ string $>$ & ${ }^{*}\left[\right.$ unassigned] ${ }^{*}$ & Required processor speed \\
\hline ProcessorArchitecture & $<$ string $>$ & ${ }^{\prime \prime}[$ unassigned]" & Required processor architecture \\
\hline ProcessorType & $<$ string $>$ & ".[unassigned]" & Required processor type \\
\hline ProcessorPropertyName & $<$ string $>$ & ".[unassigned]" & Required processor property \\
\hline ProcessorPropertyValue & $<$ string $>$ & ".[unassigned]" & Required processor property value \\
\hline ProcessorInternalMemorySize & $<$ string $>$ & ".[unassigned]" & Required internal device memory \\
\hline- & List $<@$ Processor $>$ & $\left.{ }^{\prime}\{\}\right\}^{*}$ & $\begin{array}{l}\text { Required processors (check Table } 2 \text { for } \\
\text { Processor details) }\end{array}$ \\
\hline MemorySize & $<$ string $>$ & ".[unassigned]" & Required memory size in GBs \\
\hline MemoryType & $<$ string $>$ & ".[unassigned]" & $\begin{array}{l}\text { Required memory type (SRAM, } \\
\text { DRAM, etc.) }\end{array}$ \\
\hline StorageSize & $<$ string $>$ & ".[unassigned]" & Required storage size in GBs \\
\hline StorageType & $<$ string $>$ & ."[unassigned]" & $\begin{array}{l}\text { Required storage type (HDD, SSD, } \\
\text { etc.) }\end{array}$ \\
\hline OperatingSystemType & $<$ string $>$ & ".[unassigned]" & $\begin{array}{l}\text { Required operating system type } \\
\text { (Windows, MacOS, Linux, etc.) }\end{array}$ \\
\hline OperatingSystemDistribution & $<$ string $>$ & ".[unassigned]" & $\begin{array}{l}\text { Required operating system distribu- } \\
\text { tion (XP, Sierra, openSUSE, etc.) } \\
\end{array}$ \\
\hline OperatingSystemVersion & $<$ string $>$ & ".[unassigned]" & Required operating system version \\
\hline WallClockLimit & $<$ string $>$ & ".[unassigned]" & Maximum wall clock time \\
\hline HostQueues & $<$ string $>$ & "[unassigned]" & Required queues \\
\hline AppSoftware & $<$ string $>$ & ".[unassigned]" & $\begin{array}{l}\text { Required applications that must be } \\
\text { available within the remote node for } \\
\text { the task }\end{array}$ \\
\hline
\end{tabular}


All constraints are defined with a simple value except the HostQueue and AppSoftware constraints, which allow multiple values.

The processors constraint allows the users to define multiple processors for a task execution. This constraint is specified as a list of @Processor annotations that must be defined as shown in table 2

\begin{tabular}{|c|c|c|l|}
\hline Annotation & Value type & Default value & Description \\
\hline computingUnits & $<$ string $>$ & ${ }^{\prime} 1^{*}$ & $\begin{array}{l}\text { Required number of computing } \\
\text { units }\end{array}$ \\
\hline name & $<$ string $>$ & ${ }^{*}[\text { unassigned }]^{*}$ & Required processor name \\
\hline speed & $<$ string $>$ & ${ }^{*}[\text { unassigned }]^{*}$ & Required processor speed \\
\hline architecture & $<$ string $>$ & ${ }^{*}[\text { unassigned }]^{*}$ & $\begin{array}{l}\text { Required processor architec- } \\
\text { ture }\end{array}$ \\
\hline type & $<$ string $>$ & ${ }^{*}[\text { unassigned }]^{*}$ & Required processor type \\
\hline propertyName & $<$ string $>$ & ${ }^{*}[\text { unassigned }]^{*}$ & Required processor property \\
\hline propertyValue & $<$ string $>$ & ${ }^{*}[\text { unassigned }]^{*}$ & $\begin{array}{l}\text { Required processor property } \\
\text { value }\end{array}$ \\
\hline internalMemorySize & $<$ string $>$ & ${ }^{*}[\text { unassigned }]^{*}$ & $\begin{array}{l}\text { Required internal device mem- } \\
\text { ory }\end{array}$ \\
\hline
\end{tabular}

Table 2: Arguments of the @Processor decorator

\subsubsection{Implement decorator}

PyCOMPSs allows the user to define several versions of the same tasks. The main idea behind this functionality is having several ways to perform the same operation. In the general case, the constraints of each version are different.

For example, it would be possible to have an implementation for an accelerator (GPU or FPGA) and an implementation using common CPUs. It would also be possible to define an implementation that uses OpenMP occupying a single node and a MPI version occupying several computing nodes.

Figure 3 a use example of this decorator. In this case, the secondary implementation uses a library that may be installed in a subset of the computing nodes. The information that must be provided is the class in which the main implementation is coded and the method name.

\subsection{HyperLoom functionalities}

\subsubsection{Dynamic resource requests}

HyperLoom allows to define resource request for each task individually. 


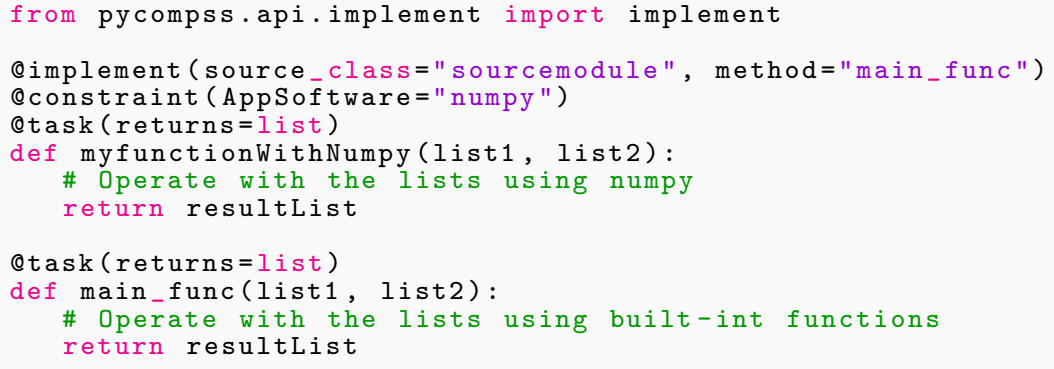

Figure 3: Implement usage example

\subsubsection{Run external program with zero-copy execution}

HyperLoom provides "run" method to running external programs. Data objects are managed via symlinking and moving on RAM disk. It avoids unnecessary copy of data and reading data by worker at all if computation stays on the same worker.

\section{Methods}

Both frameworks have to implement all the decorations and API calls described in this section even if they don't have any impact in the scheduling process. This fact guarantees that the code do not crash when switching between them. It has to be taken into account that Figures 4 and 5 contain the wrapper coded to work in local. This code helps understanding the expected behavior but does not have any scheduling implementation.

\subsection{Decorators}

\subsubsection{Task decorator}

First of all, a decorator has been defined to indicate the tasks. It's basic syntax is shown on Figure 4. All the functions marked with this decorator will be executed remotely. By default it is assumed that all the function parameters are IN and there is no return value. In addition, there are two hints that must be given otherwise:

1. returns allows the user to say how many return values has the function.

2. variable_name $=\{$ INOUT, FILE_IN, FILE_OUT, FILE_INOUT $\}$ makes possible to indicate either that the input object is modified or that the input string corresponds to a file. This way, the scheduler can transfer the file between the diferent worker nodes to make the execution possible.

Finally, it has to be taken into account that there is an optional parameter associated to the keyword scheduling_constraints that makes possible to pass an instance of ExaquteExecutionConstraints that allows the user to specify task constraints in a dynamic way. That is, in execution time. This point has to be discussed more deeply considering the users feedback. Even if it allows them more expresivity, introduces scheduling dependent code into de user's code, fact that was agreed to avoid at the maximum. Alternatively, it would be possible to define several versions of the same call with different execution constraints. 


\subsubsection{Constraint decorator}

Considering that this is a functionality that BSC had already implemented, the syntax remains exactly as presented on subsection 2.2. More keywords could be added considering the user's demands and the project's particularities.

\subsubsection{Implements}

This case is analogous to the previous point. Hence, the same notation already implemented on PyCOMPSs has been kept.

\subsection{API calls}

Figure 5 shows the basic definition of the api calls implemented. The following points summary their basic expected behavior:

- barrier()

The purpose of this call is to wait until all the tasks have been executed.

- get_value_from_remote(obj)

This API call brings back to the master/client the value of a remote computed object.

- delete_object (obj)

This API call removes all the copies allocated in the workers of the given object.

- delete_file(path_to_file)

This API call removes all the copies allocated in the workers of the given file.

- compute(value)

This API call submits the given value to the scheduler, starting the computation considering all the DAG generated until the moment.

\section{Example of usage}

This example, even is is a toy code that does not perform any useful computation, has the property of testing all the functionalities described in section 3. Figure 6 shows how the example code launches the initialization of several models with really variate granularity in distributed nodes. This generated model is defined in Figure 8. The most important think to realize that it contains the Kratos model that will have to be handled in the real project. In addition, an intermediate class has been defined as shown in Figure 7 to show how distributed computations can be hidden in an intermediate class to ease the workflow definition. All the distributed functions have been defined in Figure 7 to ease the identification of the parts that will be executed in the worker nodes. The interactions with the scheduler are completelly contained in this file. Hence, changing from local to distributed and between both frameworks is done changing a single import. 


\section{Data requirements}

Concerning the data requirements, it is possible to define two different project phases. Initially, data will be serialized to a single string. Next, distributed objects will be handled.

\subsection{Single serialized object}

In the first stage, medium size tasks will be launched in such a way that all the data can be fit in the memory of a single node. All the data passed to a task as a parameter must be serializable. This fact implies the definition of the following functions:

1. save

It is possible to define this functions in two different ways.

First of all, the function could have the following parameters:

- obj: input object to serialize

- return: a string or a stream in which to write the serialized object

Otherwise, an alternative definition is also possible:

- obj: input object to serialize

- path: input string representing the path into which a file containing the serialized object should be created

2. load

Afterwards, the serialized objects must be recoverable. This implies providing the symmetrical functionalities to the ones defined previously.

In case the first option has been chosen, the following parameters are involved into the function call:

- str: input string or stream containing the serialized object

- return: original object passed to the save function

The second version of this function should have the following parameters:

- path: input string representing the path of the file containing the serialized object

- return: original object passed to the save function

\subsection{Distributed serialized object}

In a more advanced stage, mpi simulations will be run, occupying several computing nodes. The case in which a single node memory is not capable to store all the data handled by each task is contemplated. Hence, a single serialized string/file is no longer possible. There is a deliverable in the 18th month concerning this problematic. Nevertheless, the discussion has already started. Some desirable properties have already been defined:

- The objects should be serialized in a distributed way, meaning that each MPI process should be responsible to the serialization and later recovery of its piece of data 
- Since there is no longer a single serialization for each object, two main strategies are possible to express the dependencies between tasks:

1. Define a component into the programming model that can be directly called from the mpi processes in such a way that it tracks all the serialized model parts and its location, being in charge to store it in a file in the early stages and in memory in more advanced phases

2. Not considering the whole model at all and define all the dependencies in function of the several parts. In this case, the scheduler should be aware of the MPI process associated to each dependency in order to perform the transferences and set all the MPI environment variables accordingly.

Both solutions succeed in avoiding the shared file system and the serialization of the whole model into a single serialization. However, the first option seems more interesting as it delegates all the data handling part to the programming models. It keeps better the philosophy of having as less code as possible related to its distribution.

Nevertheless, it is important to keep in mind that this phase is still in a really early stage. Some discussion between all the involved partners needs to be carried out. A consensus should be achieved in such a way that the desired properties are achieved and the solution fits as well as possible in the already implemented codes. 


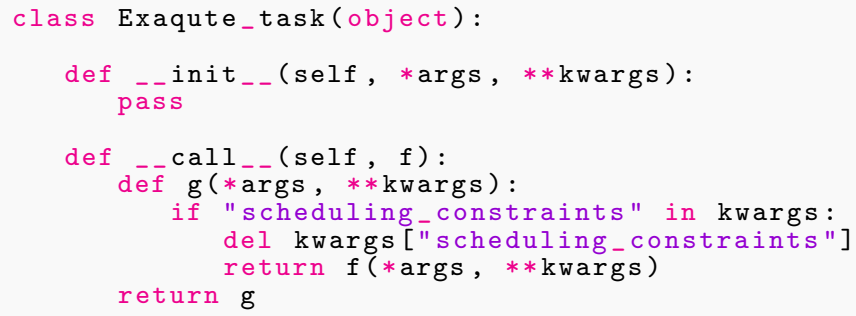

Figure 4: Decorator definition for the local case

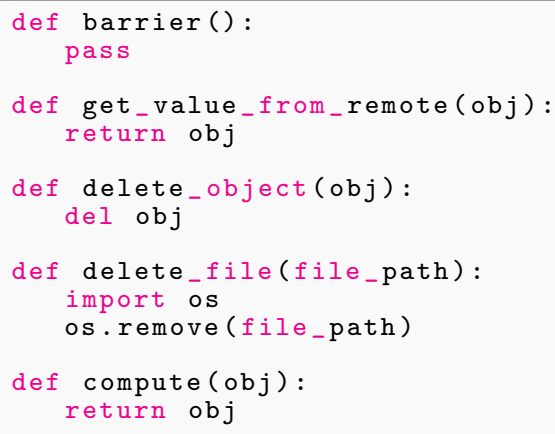

Figure 5: API calls definition for the local case

\section{References}

A Methods

B Example of usage 


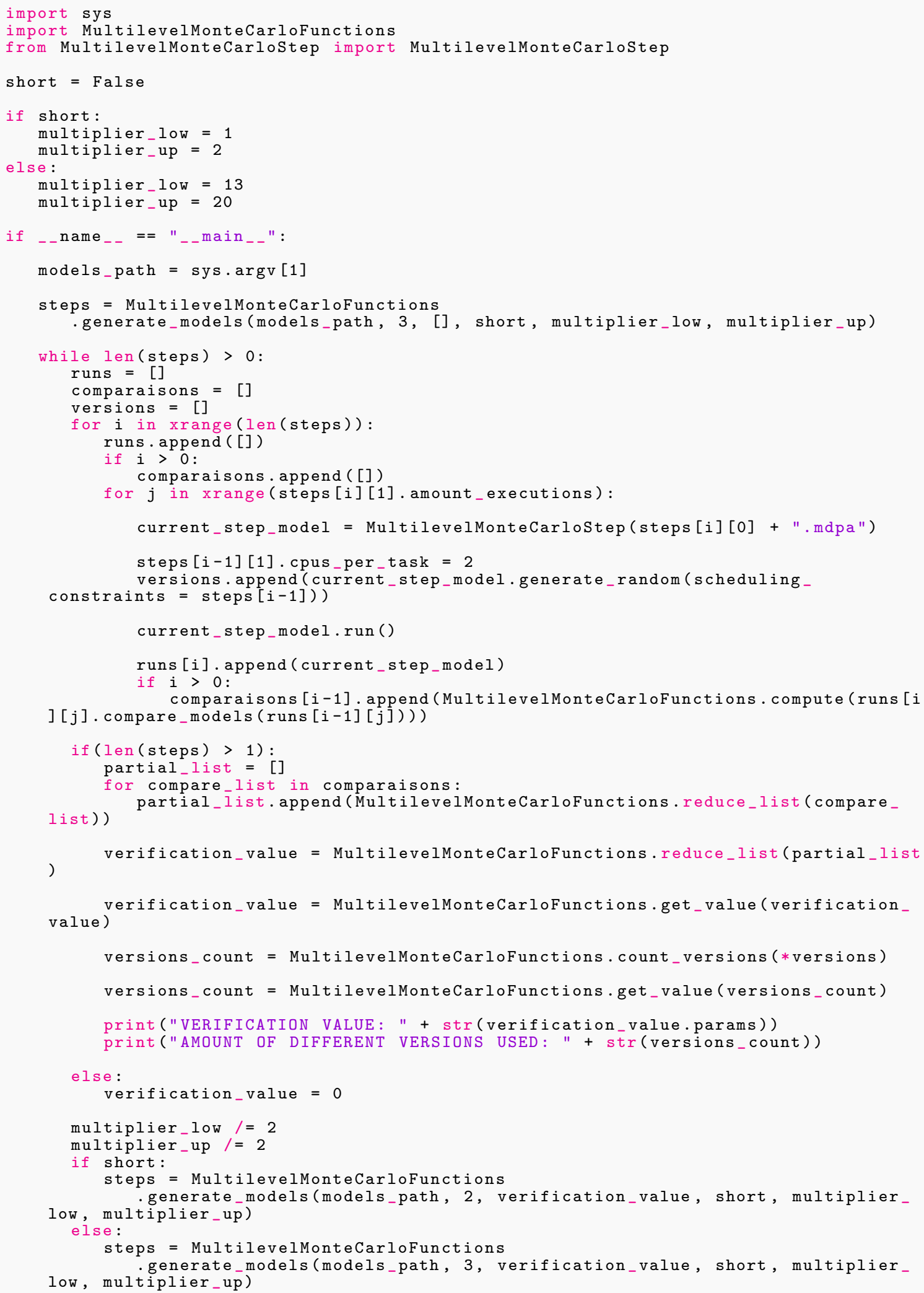

Figure 6: Basic use example 


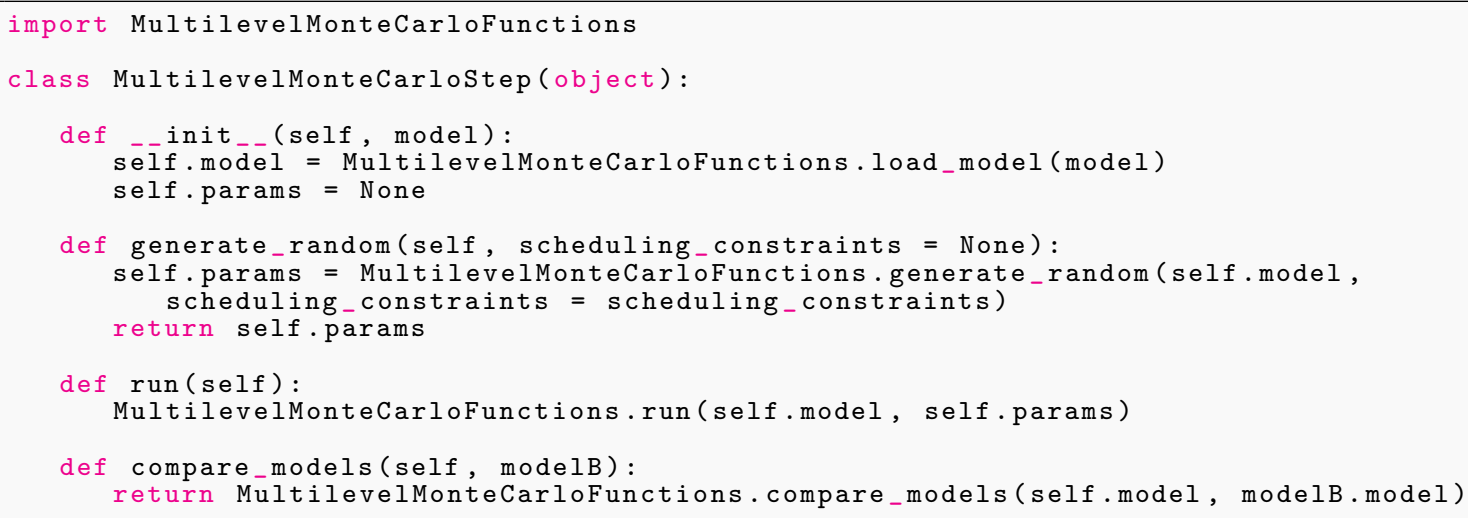

Figure 7: Monte Carlo step definition

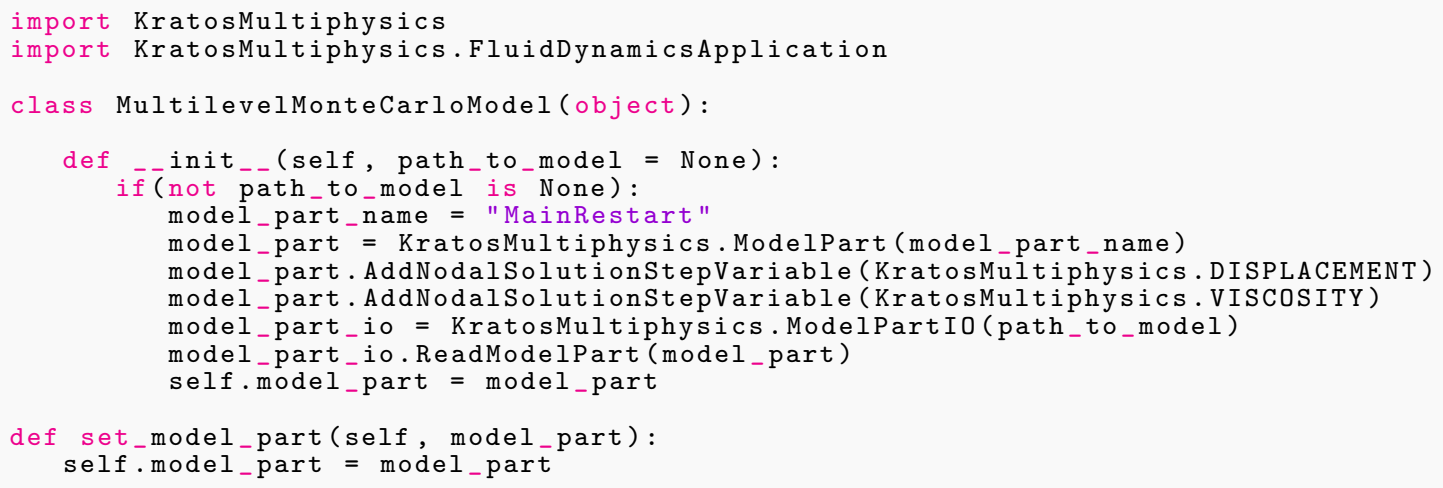

Figure 8: Model definition 


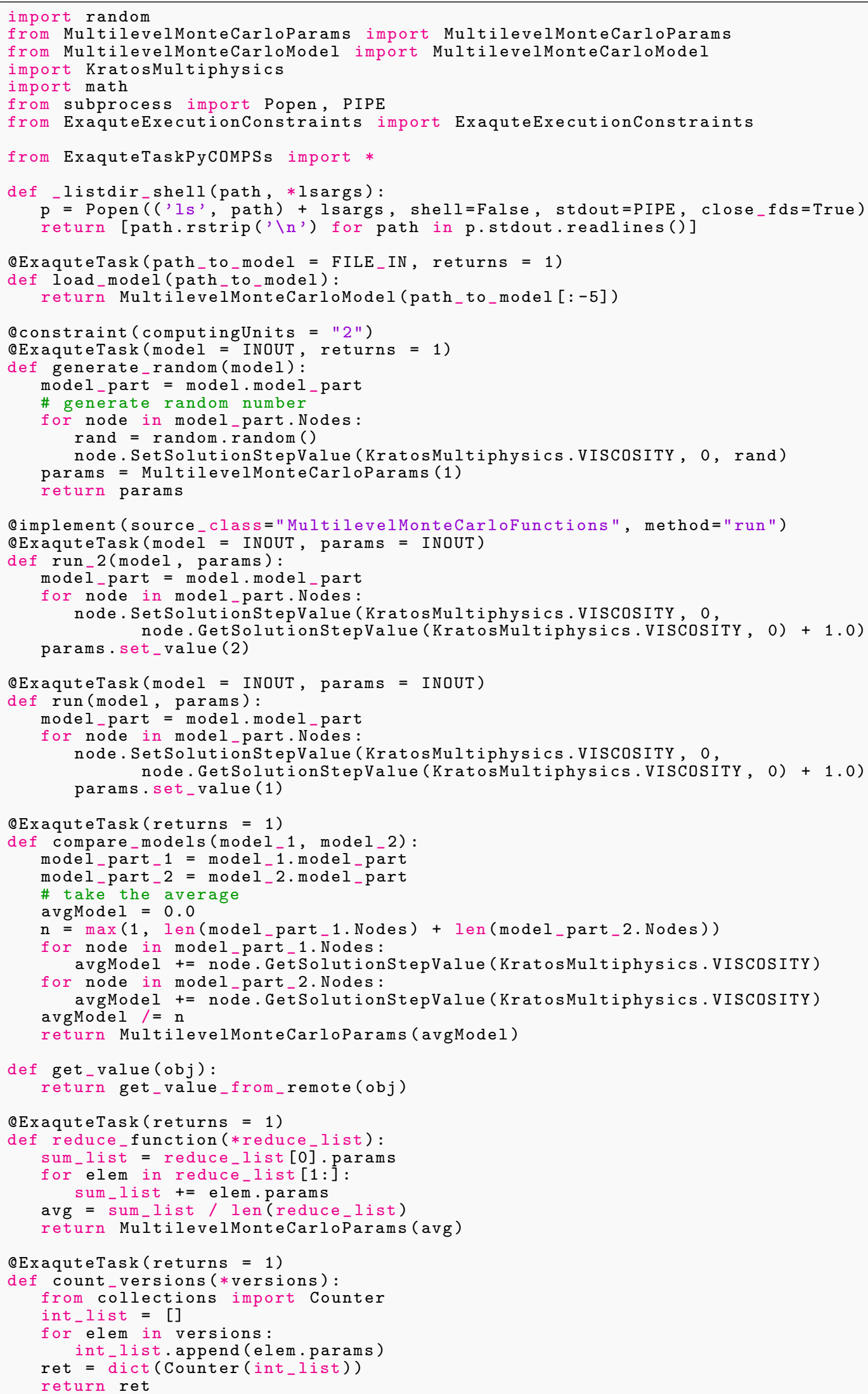

Figure 9: Function definition 